\title{
Fusion of Laserscannner and Video Based Lanemarking Detection for Robust Lateral Vehicle Control and Lane Change Maneuvers
}

\author{
Florian Homm, Nico Kaempchen and Darius Burschka
}

\begin{abstract}
The knowledge about lanes and the exact position on the road is fundamental for many advanced driver assistance systems. In this paper, a novel iterative histogram based approach with occupancy grids for the detection of multiple lanes is proposed. In highway scenarios, our approach is highly suitable to determine the correct number of all existing lanes on the road. Additionally, the output of the laserscannner based lane detection is fused with a production-available vision based system. It is shown that both sensor systems perfectly complement each other to increase the robustness of a lane tracking system. The achieved accuracy of the fusion system, the laserscannner and video based system is evaluated with a highly accurate DGPS to investigate the performance with respect to lateral vehicle control applications.
\end{abstract}

\section{INTRODUCTION}

The information about the position of the vehicle on the road is the basis for several applications. In particular, for highly automated driver assistance systems, such as the BMW emergency stop assistant [1], it is fundamental to have an accurate and robust in-lane localization to derive the parameters required by a lateral vehicle controller, but also to have detailed information about the position and number of adjacent lanes to enable complex driving strategies, such as lane change maneuvers.

A popular approach is the detection of longitudinal lane markings. Once they have been extracted, it is assumed that they define the drivable path on the road and include the overall important information about the lanes. In the past decades, extensive research has been done in the field of lane detection. Most authors use vision to extract lane markings. Today, vision based algorithms perform very well even in difficult situations (inconsistent texture of the road surface or inconspicuous markings) and provide stable results which can be used for lateral vehicle control. A comprehensive overview can be found in [2], [3]. However, all vision based approaches suffer the typical problems of passive camera systems. Under certain situations, for instance during dynamic changes of ambient brightness, direct sunlight or shadow patterns on the road surface, the detection of lane markings using vision can be extremely difficult [3]. Furthermore, the limited horizontal field of view of automotive video sensors makes it almost impossible to detect neighboring lane markings, which are far away from the ego vehicle. Unlike video sensors, laserscanners actively emit a signal

F. Homm is with BMW Group, Research and Technology, D-80788 Munich, Germany florian. homm@bmw. de

N. Kaempchen is with BMW Group, Research and Technology, D-80788 Munich, Germany nico. kaempchen@bmw. de

D. Burschka is with Technische Universität München, Department of Computer Science, D-85748 Garching, Germany burschka@cs.tum. edu and operate by the measurement principle of time-of-flight. Todays automotive laserscanners have a wide horizontal field of view and multiple vertical layers to compensate for pitch motions. Some of these vertical layers scan the road surface in front of the vehicle and detect targets right above or on the ground. While in-vehicle camera sensors provide dense measurements for lanemarking feature extractions, automotive laserscanners only yield quite sparse measurements from a flat observation angle (Fig. 1). Hence, one of the key challenges in laserscannner based lane detection is to extract the measurements which are caused by the lane markers (signal) and distinguish them from the measurements created by the road pavement (noise). Previous work done to laserscannner based lane detection are [4], [5], [6], [7] and [8]. However, none of these approaches combine both the reliable detection of parameters required by a lateral vehicle controler - such as the offset, heading and curvature of the lane- and the detection of neighboring lanes. In this paper, a novel iterative histogram based approach with occupancy grids for the detection of multiple lanes is proposed. The algorithm performs a global optimization and can deal with sparse and noisy measurements to provide lane information even at maximum noise levels. Furthermore, it is shown how to utilize a spectral analysis in the frequency domain to distinguish between solid and dashed road markings to obtain a consistent road model. Additionally, the output of the laserscannner based lane detection is fused with a production-available vision based system. It is shown that both sensor systems perfectly complement each other to achieve a robust and continuous lane detection.

\section{LASERSCANNNER BASED LANE DETECTION}

In this section, a brief introduction to the measurement principle of laserscannner based lane marking detection is first given. Then, the computation of an occupancy grid to model and track arbitrary laserscannner ground reflection measurements is discussed. In the main part, a global iterative histogram optimization algorithm for the robust parameter extraction of lane markings is proposed. Finally, it is shown how to build a consistent road model from extracted lane markings.

\section{A. Measurement Principle}

Unlike cameras, laserscanners do not directly measure the brightness contrast between the white or yellow lane markings and the road pavement. As previously mentioned, they actively emit a signal and record its echo. Whether an echo is returned or not, depends on a variety number of properties. For instance such as the distance to the target, the 
surface material and the angle between the surface normal and the beam. In case of a road surface the perspective observation angle (Fig. 1) is rather flat and for an arbitrary surface any combination of diffuse or specular reflection may occur and lead to light reflections emitting in all directions. This makes it extremely difficult for laserscanners to retrieve a signal which is above the noise level. Fortunately, in order

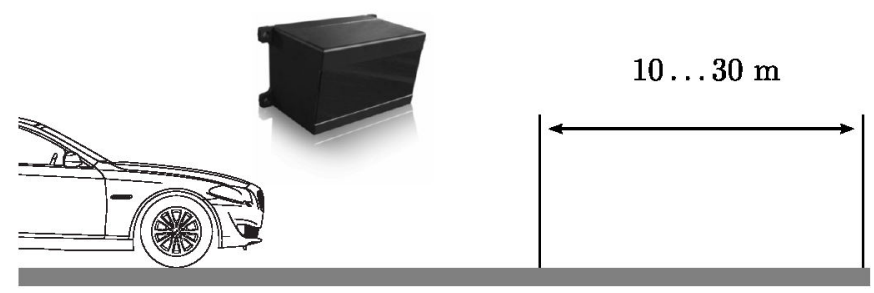

Fig. 1. Lanemarking detection with a 4 layer laserscannner. The lower two layer scan the road surface at a distance of $10 \ldots 30 \mathrm{~m}$.

for lane markings to be seen by drivers at night, they must be retroreflective [9], [10]. In case of retroreflective lane markings, incoming light from vehicle headlamps is reflected back towards the vehicle. This principle is analogue for light detection and ranging devices, such as laserscanners. For permanent road markings retroreflectivity is achieved by embedding glass beads in the marking material (Fig. 2). Hence, if a measurement on the ground is detected, it is likely that it occurred from a white or yellow road marking.

Another feature of modern laserscanners that can be exploited to increase the number of measurements on possible road markings is the so called multi-echo or multitarget measurement feature [11]. This feature describes the capability to detect multiple targets at various distances within a single shot. For flat observation angles the beam is spreaded over a wide area on the road surface and in the case of a lane marking the light is reflected from multiple points on the marking. The device used in our system was a 4 layer automotive laserscannner with a horizontal opening angle of $100^{\circ}$ and a vertical opening angle of $3.2^{\circ}$. This device comes with a multi-target feature to detect up to 3 targets within a single beam, and a built-in ground classification to distinguish between objects (obstacles) and ground measurements (road surface). Mounted behind the front bumper, the lower two layers scan the road surface at a distance of $10 \ldots 30 \mathrm{~m}$ (Fig. 1).

\section{B. Occupancy Grid Based Environment Mapping}

In general, a map of the environment can be seen as a list of objects in the environment with their locations. Occupancy grids [12] are a location-based representation of the environment and are the first choice if the geometry of objects can be quite complex and hard to describe by geometric primitives. They divide the environment into an evenly spaced grid of cells and estimate the probability of each cell being occupied, based on the sensor readings. Let $m$ be a planar occupancy grid. A single grid cell is denoted as $m_{x, y}$ and $z_{1}, \ldots, z_{t}$ denotes all of the measurements up to time $t$ along with the vehicle poses $x_{1}, \ldots, x_{t}$. Each grid

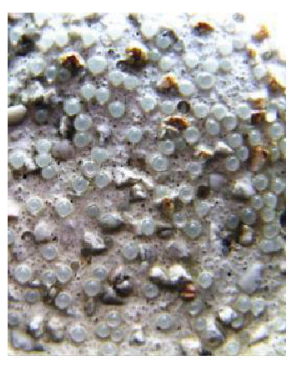

(a)

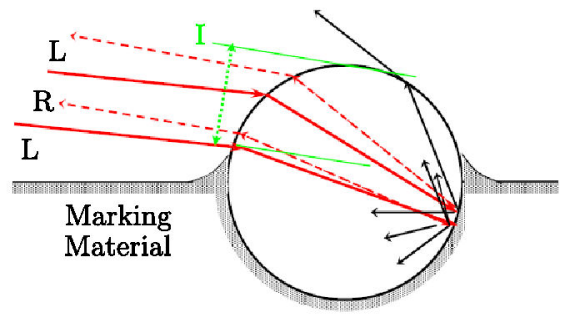

(b)
Fig. 2. In (a), microscopic picture of white marking material with embedded glass beads. In (b), working principle of retroreflective glass beads [10].

cell corresponds to a binary value which specifies whether a cell is occupied or not. The notation $p\left(m_{x, y}=1\right)$ is used to denote an occupied cell. Furthermore, it is assumed that $x_{1}, \ldots, x_{t}$ are known which is called mapping with known poses [12]. The problem addressed by occupancy grid mapping is to determine the posterior

$$
p\left(m \mid z_{1}, \ldots, z_{t}, x_{1}, \ldots, x_{t}\right)
$$

given all measurements and poses. However, grid maps are defined over high-dimensional space and this posterior cannot be computed easily. The classical occupancy grid algorithm breaks down the problem into many one-dimensional estimation problems, which are treated independently of each other. The problem is reduced to the estimation of the posterior for each $m_{x, y}$ of the grid:

$$
p\left(m_{x, y} \mid z_{1}, \ldots, z_{t}, x_{1}, \ldots, x_{t}\right)
$$

After transformation to the so called log odds form, applying Bayes' Theorem and removing some hard-to-compute terms, we finally get:

$$
\begin{aligned}
\frac{p\left(m_{x, y} \mid z_{1}, \ldots, z_{t}\right)}{1-p\left(m_{x, y} \mid z_{1}, \ldots, z_{t}\right)}= & \frac{p\left(m_{x, y} \mid z_{t}\right)}{1-p\left(m_{x, y} \mid z_{t}\right)} \\
& \cdot \frac{p\left(m_{x, y} \mid z_{1}, \ldots, z_{t-1}\right)}{1-p\left(m_{x, y} \mid z_{1}, \ldots, z_{t-1}\right)}
\end{aligned}
$$

A detailed description on how to efficiently compute grid maps and model occurring measurement errors can be found in [13]. Fig. 3 shows an example of three different occupancy grids computed with pre-classified sensor readings. The fact that there are just a few sensor readings which belong to valid road markings and the fact that the grid can be heavily perturbed by additive noise makes robust lane marking detection with laserscanners an extremely challenging task.

In the following section, a global iterative histogram optimization algorithm for a robust parameter extraction of lane markings is proposed.

\section{Histogram-Based Parameter Optimization}

In lane detection, one is mainly interested in an abstract representation of the road that can be directly used as input for a controller or a fusion system. Like in [6], a circular model to describe the road is used. This model comes with the minimum set of parameters to approximate a road which 


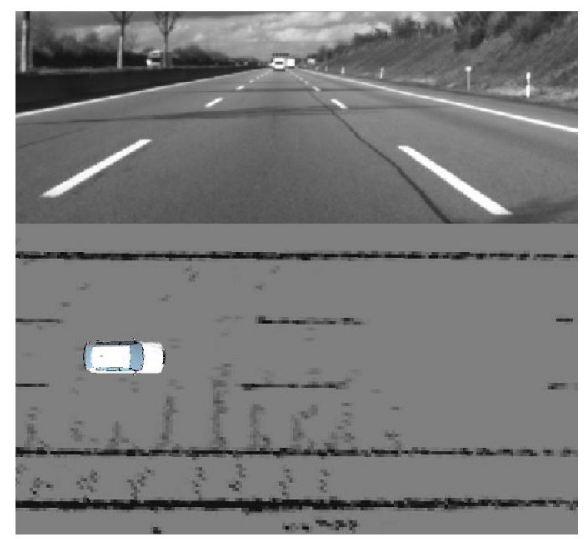

(a)

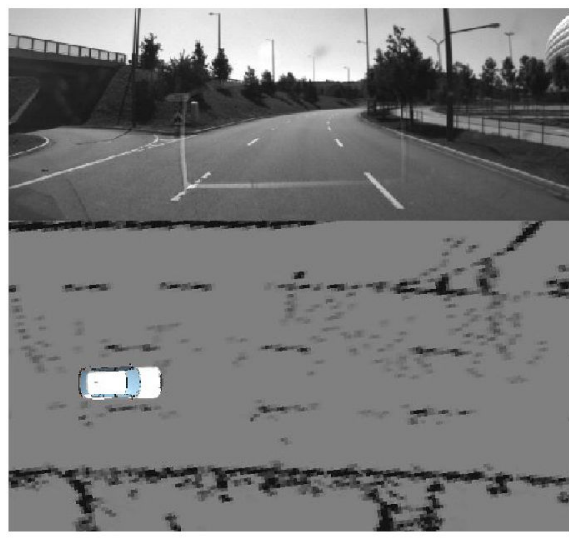

(b)

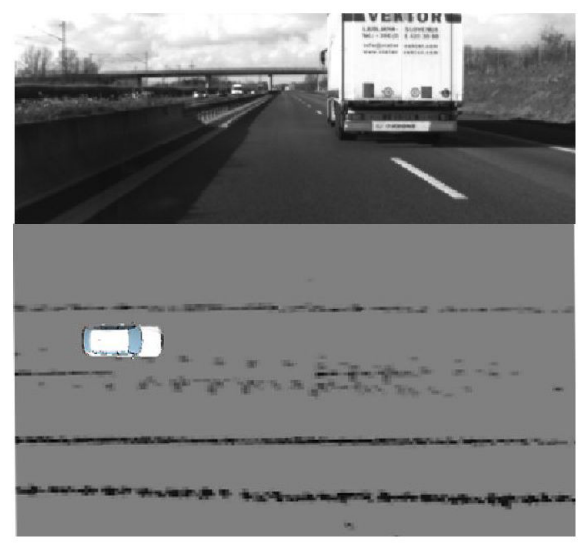

(c)

Fig. 3. Example for three different occupancy grids computed with pre-classified sensor readings. In (a), additive noise induced by pavement reflections. In (b), noise induced by pitch movements and surface irregularities. In (c), misclassified ground points in a leading vehicle. The cell size was set to $0.2 \mathrm{~m}$.

consists of lines, clothoids and circles [14]. Each lane can be uniquely described by the state vector:

$$
f_{i}=\left[\begin{array}{llll}
c_{i} & \gamma_{i} & y_{i} & w_{i}
\end{array}\right]^{T}
$$

where $c$ is the curvature, $\gamma$ the heading angle, $y$ the offset to the center of the lane and $w$ the width of the lane. The set of all existing lanes is called a road.

$$
S=\left\{f_{1}, \ldots, f_{m}\right\}
$$

The major goal of the histogram-based parameter extraction is to find all existing lanes $f_{i}$ in the occupancy grid $m$. Let

$$
P_{\mathrm{S}}=\left\{f_{0}, \ldots, f_{i}, \ldots, f_{n}\right\}
$$

be the set of all possible parameter combinations. There exists a subset $S \subset P_{\mathrm{S}}$ that includes all existing lanes in $m$. The extraction of this subset is not trivial and to reduce the complexity of the search space it is assumed that the heading $\gamma$ and curvature $c$ of all lane markings in $m$ is equal. This directly implies parallel non-overlapping lane marking and is a valid assumption for roads without crossings.

$$
\begin{aligned}
& \overline{f_{i}}=\left[\begin{array}{llll}
\bar{c} & \bar{\gamma} & y_{i} & w_{i}
\end{array}\right]^{T} \\
& S=\left\{\overline{f_{1}}, \ldots, \overline{f_{n}}\right\}
\end{aligned}
$$

The key idea of the proposed algorithm is to represent $S$ as a corresponding set of histograms over $m$ where each variation of $c_{k}$ and $\gamma_{l}$ defines a single histogram $H_{i}$. Each bin $k_{i}$ contains the normalized accumulated sum over the probabilities $p(x, y)$ in $m$ along a circular path $K_{r}$ with curvature $c_{k}$ oriented by heading $\gamma_{l}$ (9). The position of each bin in $H_{i}$ is given by a discrete offset $y$ along a straight line $g\left(\alpha_{l}, x, x_{0}, y_{0}\right)$ perpendicular to the circular path with $\alpha_{l}=\gamma_{l}+\frac{\pi}{2}$ (Fig. 4). For practical reasons, the discretization steps of $y$ should equal the cell resolution of the corresponding grid.

$$
k_{i}\left(c_{k}, \alpha_{l}\right)=\sum_{x=-j}^{j} f\left(y, x, c_{k}, \alpha_{l}\right)
$$

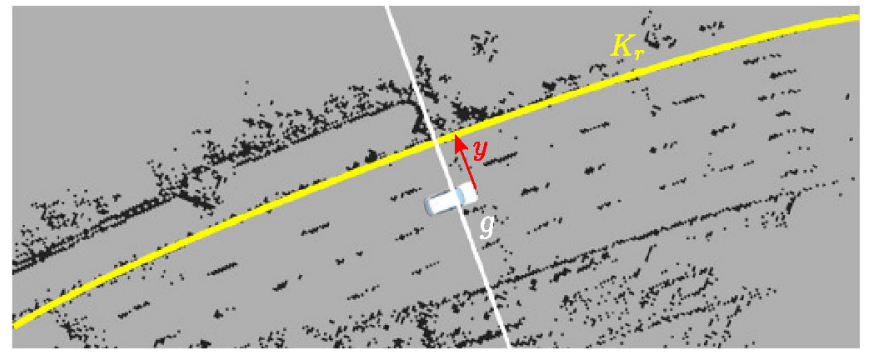

Fig. 4. Example for a circular Path $K_{r}$ with offset $y$ on the straight line $g$. The normalized accumulated sum along $K_{r}$ corresponds to one single bin $k_{i}$ in $H_{i}$.

For instance, if $c_{k}=\gamma_{l}=0$ the circular path binning is equivalent to a row or column wise histogram binning. A histogram $H_{i}$ is denoted as optimal histogram $H_{\mathrm{opt}}$, if there exists no other parameter variation of $c_{k}$ and $\gamma_{l}$ in $P_{\mathrm{S}}$, that gives a better approximation of the existing lane markings in $m$. Fig. 5(a) shows the corresponding optimal histogram to the occupancy grid in Fig. 4. Fig. 5(b) and 5(c) outline what happens to the histogram $H_{o p t}$ if a small error is added to the curvature $c$. With an increased error in the curvature

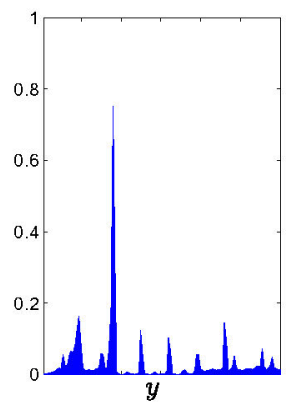

(a)

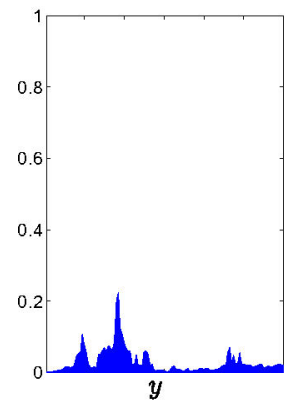

(b)

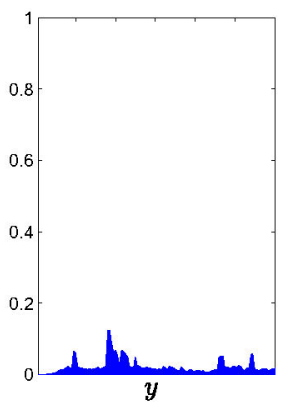

(c)
Fig. 5. In (a), optimal histogram $H_{\text {opt }}$ with $c=-0.00076 \mathrm{~m}^{-1}$. In (b), an additive curvature error of $0.002 \mathrm{~m}^{-1}$. In (c), $0.006 \mathrm{~m}^{-1}$.

of the circular path, the histogram $H_{\text {opt }}$ degenerates to a flat uniform distribution. This is because lane markings in $m$ now 
distribute among multiple bins of $H_{i}$ instead of falling into a specific bin $k_{i}$. Similarly for $\gamma$, each deviation of the optimal heading parameter causes a degeneration of the histogram $H_{i}$. This fact can be exploited to define a cost function $q\left(H_{i}\right)$ which rates each histogram according to its distribution.

$$
q\left(H_{i}\right)=\sum_{i=-d}^{d} k_{i}\left(c_{k}, \alpha_{l}\right)^{2}
$$

The sum over the squares of $k_{i}$ is used to give a higher weight to histograms which have all probabilities distributed in a minimum set of bins. Fig. 6 shows the results of the cost function (10) for different variations of the curvature and the heading. It is visible that there exists a maximum $q\left(H_{\text {opt }}\right)$

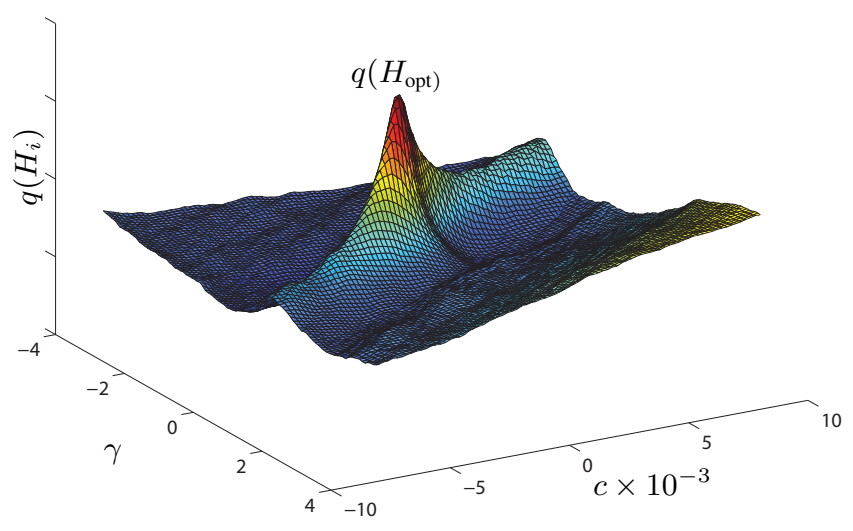

Fig. 6. Results of the cost function $q\left(H_{i}\right)$ for $\gamma=-4^{\circ} \ldots 4^{\circ}$ and $c=$ $-0.0001 \ldots 0.0001 \mathrm{~m}^{-1}$

which represents the optimal parameter combination of $c_{k}$ and $\gamma_{l}$. Thus, the problem to find the heading and curvature of the road becomes a non-linear parameter optimization problem. Due to the computational burden, it is not feasible to evaluate the cost function $q$ over all possible combinations of $c_{k}$ and $\gamma_{l}$. A modified version of the iterative NelderMead simplex optimization algorithm [15] is used to obtain the optimal histogram $H_{\text {opt }}$. At each iteration, the algorithm computes a set of 3 histograms (parameter space plus one) in a simplex order and rates them according to the cost function (10). The Simplex algorithm is fast, deterministic and robust to local minima. After a sufficient number of iterations the Simplex algorithm converges to the maximum and remains stable and a total number of 5-8 iterations at each timestep is sufficient to keep track of the curvature and heading of the road. In order to allow the optimizer to evaluate a new parameter combination after it has converged to the maximum, the simplex has to be widened after each new measurement or position update. Once the optimal histogram has been estimated, single lanes can be extracted from $H_{\text {opt }}$. This entire process can be divided into three main parts: Local maxima detection, single marking classification and checking for road constraints.

1) Local Maxima Detection in the Histogram: In order to identify the indices $i$ of the bins which are likely to represent the position of an existing lane marking, each bin $k_{i}$ has to be compared with a threshold function. Since $H_{\text {opt }}$ can be perturbed by local random noise, a simple global threshold function is not suitable. Furthermore, different types of lane markings (solid or dashed) can have different heights of bins and have to be treated separately when using a global threshold. Therefore, a signal-to-noise-ratio (SNR) for each bin $k_{i}$ in a predefined vicinity $u \ldots s$ is used to generate valid lane marking hypothesis. We define the SNR of a single bin $k_{i}$ as:

$$
\begin{aligned}
v_{l} & =\max \left(k_{-u}, \ldots, k_{-s}\right) \\
v_{r} & =\max \left(k_{s}, \ldots, k_{u}\right) \\
\operatorname{SNR}\left(k_{i}\right) & =20 \lg \left(\frac{k_{i}}{\max \left(v_{l}, v_{r}\right)}\right)
\end{aligned}
$$

If the SNR of a specific bin $k_{i}$ is above a certain threshold, $k_{i}$ is accepted as a valid marking hypothesis. Furthermore, once the single peaks have been identified we can interpolate between $k_{i-1}, k_{i}$ and $k_{i+1}$ to obtain an accuracy for the offset which is higher than the previously chosen cell resolution of $m$. Since the accumulated measurements in $m$ are Gaussian, $H_{\mathrm{opt}}$ is Gaussian too and a performed $s u b$ pixel computation between adjacent bins (circular paths) can improve the accuracy by a factor of 2-3.

2) Single Marking Classification and Checking for Road Constraints: Since not all marking hypotheseses belong to valid road markings, it is necessary to verify each single marking in the context of a global road model. As a first step, each single marking has to be classified into one of two classes: solid or dashed. All solid lane markings usually indicate no-passing zones which can be exploited to derive the road edges. This information is also of high importance to safely derive driving strategies such as lane change maneuvers.

The classification is performed by a threshold test based on spectral analysis in the frequency domain. To do so, all probabilities $p(x, y)$ which previously have been assigned to the bin $k_{i}$ are considered as a discrete signal $S$ along the corresponding circular path $K_{r}$ in $m$. This signal gives a curvature and heading compensated discrete representation of each lane marking in $m$. In an ideal case, the period of the signal $S$ conforms to a rectangle function if the marking is likely to belong to the class dashed. Otherwise, in case of a solid marking the period of $S$ is infinity. The period of the signal $S$ can be obtained from the auto-powerspectrum which is the squared absolute value of the Fourier transformed signal $S$ (Eq. 12 and 13). This approach leads to good results even in case of incomplete markings with several gaps.

$$
\begin{aligned}
F\left(\omega_{n}\right) & =\sum_{k=1}^{N} e^{-i \omega_{n} t_{k}} f\left(t_{k}\right) \\
S_{x x}\left(\omega_{n}\right) & =\left|F\left(\omega_{n}\right)\right|^{2}
\end{aligned}
$$

After the classification step, single lane markings can be assigned to lane hypotheseses by checking the geometric constraints such as the minimum and maximum width for a possible valid lane. The offset $y$ for each lane hypothesis is computed by the average of the sub-pixel corrected position 
of the bins $k_{\text {left }}$ and $k_{\text {right }}$ :

$$
y_{i}=\frac{k_{l e f t}+k_{\text {right }}}{2}
$$

Finally, the lane permutation with the highest aggregated SNR is chosen as the valid road. Fig. 7 shows an example of a valid road with 3 adjacent lanes, which have been extracted from the given occupancy grid. Besides its robustness to noise, one of the key benefits of the proposed algorithm is its low computational complexity. The iterative histogram-based estimation of the lane parameters including the classification step takes less than $10 \mathrm{~ms}$ for a grid with a dimension of $256 \times 256$ cells on a Intel ${ }^{\circledR}$ Core $2^{\mathrm{TM}}$ Quad processor with $2.5 \mathrm{GHz}$

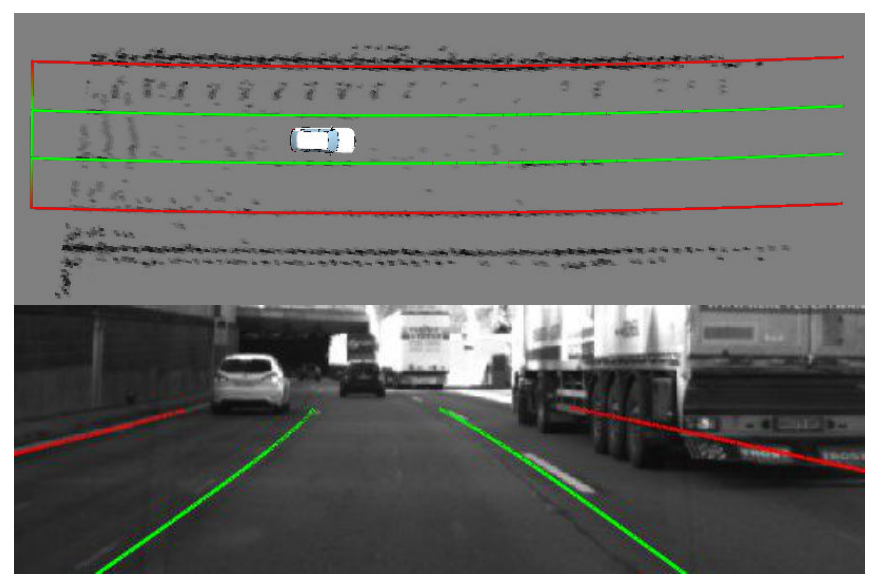

Fig. 7. Example for a valid road with 3 lanes which have been extracted from a given occupancy grid with a cell size of $0.2 \mathrm{~m}$. It can be seen that even the right lane, which is occluded in the video image, can be detected.

\section{FUSION}

In the previous section, a novel histogram based algorithm for the detection of multiple lanes was introduced. In this section, the output of the laserscannner based lane detection is fused in a high-level fashion with the parameter output of a state-of-the-art in production available vision based system for lane detection. The main goal of this fusion is to significantly improve the robustness of a lane tracking system where a single sensor system might fail because of its sensor characteristics.

\section{A. Parameter Fusion}

To combine the output of both systems, a standard Kalman filter [12] was used. In our case, the video based system only provides information about the ego-lane and therefore the state vector (15) follows the definition of a single lane in (7) where $c$ is the curvature, $\gamma$ the heading angle, $y$ the offset to the center of the lane and $w$ the width of the lane.

$$
\hat{\mathbf{x}}=\left[\begin{array}{llll}
y & w & \gamma & c
\end{array}\right]^{T}
$$

Using a constant velocity model, the dynamic model is given as follows:

$$
\left[\begin{array}{c}
y \\
w \\
\gamma \\
c
\end{array}\right]=\left[\begin{array}{cccc}
1 & 0 & T v & \frac{1}{2} T^{2} v^{2} \\
0 & 1 & 0 & 0 \\
0 & 0 & 1 & T v \\
0 & 0 & 0 & 1
\end{array}\right] \cdot\left[\begin{array}{c}
y \\
w \\
\gamma \\
c
\end{array}\right]+\left[\begin{array}{c}
0 \\
0 \\
-T \\
0
\end{array}\right] \cdot \dot{\Psi}
$$

where $T$ is the timestep, $v$ the velocity and $\dot{\Psi}$ the yaw rate of the ego-vehicle. In order to account for multiple lanes detected by the laserscanner, a low-pass filter to filter the width $w$ of each adjacent lane was used. Unlike the offset, it is independent of the ego-movement and does not require a dynamic model. Finally, extended lane information available from standard navigation maps including the actual number of lanes is used to remove outliers and temporary lanes, such as acceleration lanes.

\section{Results}

In the first part of this section, the output parameters of the laserscannner, video and fusion system are compared to a digital map as reference. In the second part, the ego-lane availability of all systems on highway scenarios including the total number of lanes is investigated.

\section{A. Accuracy}

The accuracy of the output parameters offset, heading and curvature has been evaluated using an inertial platform with differential GPS and a highly accurate digital map on a test track. The inertial platform provides position measurements with an accuracy of $1-3 \mathrm{~cm}$ at an update rate of $100 \mathrm{~Hz}$. Additionally, it delivers the actual orientation of the vehicle with an accuracy of $0.05-0.1^{\circ}$. To ensure a high accuracy of ground truth, the digital map has been computed virtually first and then used as a template to create the lane markings on the pavement. The test track had a total length of $2000 \mathrm{~m}$ and the average velocity was $100 \mathrm{~km} / \mathrm{h}$. Fig. 8 depicts the estimation errors of the three output parameters offset $y$, heading $\gamma$ and curvature $c$ in comparison to the digital map as ground-truth. The cell-size used for the occupancy grid was set to $0.2 \mathrm{~m}$ with a front view of $30 \mathrm{~m}$ and a back view of $15 \mathrm{~m}$. In table I the results are summarized by the root mean square error RMS in comparison to the ground truth. It

TABLE I

Estimation ERrors of the Proposed Algorithm

\begin{tabular}{c|ccc} 
& \multicolumn{3}{|c}{ RMS } \\
& $y(\mathrm{~m})$ & $\gamma\left({ }^{\circ}\right)$ & $c\left(\mathrm{~m}^{-1}\right)$ \\
\hline \hline Laser. & 0.034 & 0.06 & 0.0001 \\
\hline Video & 0.046 & 0.12 & 0.0001 \\
\hline Fusion & 0.037 & 0.07 & 0.0001 \\
\hline
\end{tabular}

can be seen that all parameters have an acceptable amount of error with respect to lateral vehicle control applications [16]. The proposed histogram based algorithm for lane detection achieves an accuracy which is comparable to the output of a video based lane detection system. In case of the offset and the heading it even outperforms the video based system.

\section{B. Availability}

Beside the accuracy, the overall availability of the parameters offset, heading and curvature is of high importance for lateral vehicle control tasks. The availability has been evaluated on several freeway sections in the area of Munich, covering a total length of $85.33 \mathrm{~km}$. Table II outlines the achieved results for the video, the laserscannner and the 


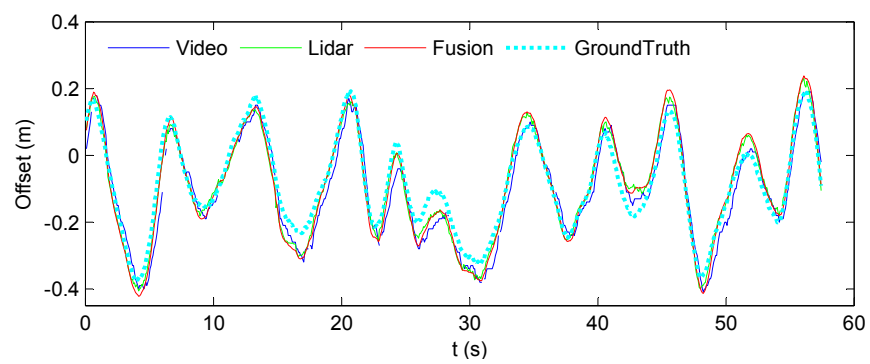

(a) Offset

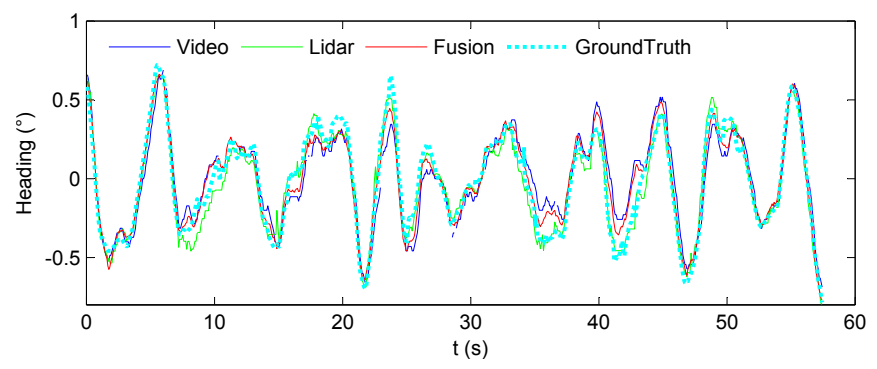

(b) Heading

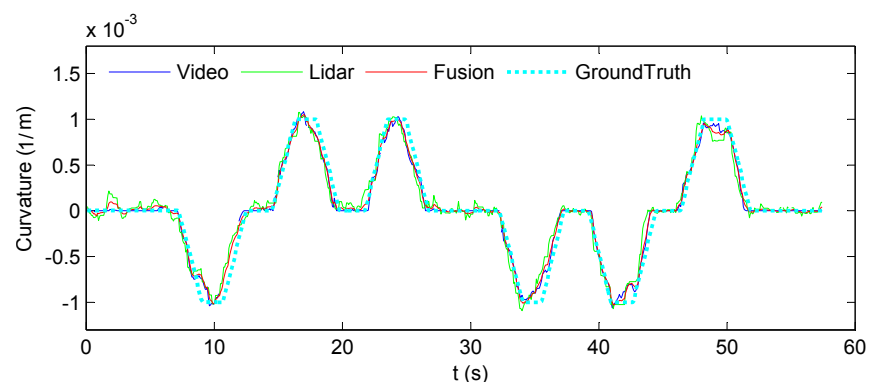

(c) Curvature

Fig. 8. Accuracy of the output parameters offset $y$, heading $\gamma$ and curvature $c$ in comparison to the digital map as ground-truth.

TABLE II

DETECTION Rate

\begin{tabular}{cc||ccc} 
Distance $(\mathrm{km})$ & Time $(\mathrm{s})$ & Video $(\%)$ & Lidar $(\%)$ & Fusion $(\%)$ \\
\hline \hline 85.33 & 2652.56 & 99.48 & 98.53 & $\mathbf{1 0 0 . 0 0}$ \\
\hline
\end{tabular}

fusion system. The video based, as well as the laserscannner based system, achieves good results with a detection rate above $98 \%$. While the failures of the video based system are mostly caused by ambient brightness changes or shadows on the road surface, the failures of the laserscanner based approach result from bad SNR ratios between the road markings and the surface. The fusion as a combination of both sensor systems achieves a detection rate of $100 \%$ which is an outstanding result. The laserscanner provides the required parameters for lateral control in cases where the the video based system has failed and vice versa. Additionally, the estimated number of lanes with respect to the feasibility of automated lane change maneuvers has been evaluated. For safety reasons, lane change maneuvers shall be only executed if all existing lanes have been detected correctly. For $97.56 \%$ of the time, the detected number of lanes was correct and in $2.44 \%$ it was less then the existing number of lanes.

\section{CONCLUSion}

A novel iterative histogram based approach with occupancy grids for the detection of multiple lanes was proposed. It has been shown that our approach provides an accuracy which is comparable to a video based system for lane detection and is highly suitable to determine the correct number of all existing lanes on the road. The robust detection of several lanes enables thus lane keeping and lane change maneuver functionalities. Furthermore, it has been proven that a video and laserscannner based system perfectly complement each other to achieve a robust and continuous lane detection.

\section{REFERENCES}

[1] Michael Ardelt, Peter Waldmann, Nico Kaempchen, and Florian Homm. Strategic decision-making process in advanced driver assistance systems. IFAC-Symposium Advances in Automotive Control, 2010.

[2] S. Sivermann and M. Trivedi. Improved video-based lane tracker performance using vehicle localization. In IEEE Intelligent Vehicles Symposium, San Diego, CA, USA, 2010.

[3] J.C. McCall and M.M. Trivedi. Video-based lane estimation and tracking for driver assistance: survey, system, and evaluation. Intelligent Transportation Systems, IEEE Transactions on, 7(1):20 -37, 2006.

[4] K. Dietmayer, N. Kämpchen, K. Fürstenberg, J. Kibbel, W. Justus, and R. Schulz. Roadway detection and lane detection using multilayer laserscanner. In Jürgen Valldorf and Wolfgang Gessner, editors, Advanced Microsystems for Automotive Applications 2005, VDI-Buch, pages 197-213. Springer Berlin Heidelberg, 2005. dietmayer.

[5] Kay Fürstenberg Jörg Kibbel, Winfried Justus. Lane estimation and departure warning using multilayer laserscanner. In Proceedings of the 8th International IEEE Conference on Intelligent Transportation Systems, 2005.

[6] A. Reyher. Lidarbasierte Fahrstreifenzuordnung von Objekten für eine Abstandsregelung im Stop\&Go-Verkehr. $\mathrm{PhD}$ thesis, Technischen Universität Darmstadt, 2006.

[7] S. Kammel and B. Pitzer. Lidar-based lane marker detection and mapping. In Intelligent Vehicles Symposium, 2008 IEEE, pages 11371142, June 2008.

[8] P. Lindner, E. Richter, G. Wanielik, K. Takagi, and A. Isogai. Multichannel lidar processing for lane detection and estimation. In Intelligent Transportation Systems, 2009. ITSC '09. 12th International IEEE Conference on, pages $1-6,2009$.

[9] Updates to research on recommended minimum levels for pavement marking retroreflectivity to meet driver night visibility needs. Technical report, U.S. Department of Transportation Federal Highway Administration. PUBLICATION NO. FHWA-HRT-07-059, 2007.

[10] DSGS-Jahrbuch Fahrbahnmarkierung 2006/2007. Technical report, Die Deutsche Studiengesellschaft für Straßenmarkierungen e.V. (DSGS), 2007.

[11] F. Ahlers and C. Stimming. Cooperative laserscanner pre-data-fusion. In Intelligent Vehicles Symposium, 2008 IEEE, pages $1187-1190$, 2008.

[12] Sebastian Thrun, Wolfram Burgard, and Dieter Fox. Probabilistic Robotics. MIT Press, 2005.

[13] F. Homm, N. Kaempchen, J. Ota, and D. Burschka. Efficient occupancy grid computation on the gpu with lidar and radar for road boundary detection. In Intelligent Vehicles Symposium (IV), 2010 IEEE, pages $1006-1013,2010$.

[14] Heiko Kramer. Modelle zur multisensoriellen Erfassung des Fahrzeugumfeldes mit Hilfe von Schätzverfahren. PhD thesis, Technische Universität Chemnitz, 2005.

[15] J. A. Nelder and R. Mead. A simplex method for function minimization. The Computer Journal, 7(4):308-313, 1965.

[16] Doris Schmidt and Hermann Winner. Kombination von fahrdynamiksenor- und globalen navigationssatellitensystemdaten zur performanceverbesserung von fahrerassistenzsystemen. In DGON Symposium Positionierung und Navigation, 2007. 